\title{
On a multi-dimensional Poissonian pair correlation concept and uniform distribution
}

\author{
Aicke Hinrichs ${ }^{2}$. Lisa Kaltenböck ${ }^{1}$. Gerhard Larcher ${ }^{1}$. \\ Wolfgang Stockinger ${ }^{3}$ (1) $\cdot$ Mario Ullrich ${ }^{2}$
}

Received: 13 September 2018 / Accepted: 23 January 2019 / Published online: 21 February 2019

(c) The Author(s) 2019

\begin{abstract}
The aim of the present article is to introduce a concept which allows to generalise the notion of Poissonian pair correlation, a second-order equidistribution property, to higher dimensions. Roughly speaking, in the one-dimensional setting, the pair correlation statistics measures the distribution of spacings between sequence elements in the unit interval at distances of order of the mean spacing $1 / N$. In the $d$-dimensional case, of course, the order of the mean spacing is $1 / N^{\frac{1}{d}}$, and-in our concept-the distance of sequence elements will be measured by the supremum-norm. Additionally, we show that, in some sense, almost all sequences satisfy this new concept and we examine the link to uniform distribution. The metrical pair correlation theory is investigated and it is proven that a class of typical low-discrepancy sequences in the high-dimensional unit cube do not have Poissonian pair correlations, which fits the existing results in the one-dimensional case.
\end{abstract}

Keywords Uniform distribution · Pair correlation of sequences · Additive energy

Mathematics Subject Classification $11 \mathrm{~K} 06 \cdot 11 \mathrm{~K} 31$

Communicated by A. Constantin.

A. Hinrichs: The author is supported by the Austrian Science Fund (FWF) Project F5509-N26, which is a part of the Special Research Program "Quasi-Monte Carlo Methods: Theory and Applications".

L. Kaltenböck, W. Stockinger, G. Larcher: The author is supported by the Austrian Science Fund (FWF), Project F5507-N26, which is a part of the Special Research Program "Quasi-Monte Carlo Methods: Theory and Applications" and Project I1751-N26.

Wolfgang Stockinger

wolfgang.stockinger@stcatz.ox.ac.uk

Extended author information available on the last page of the article 


\section{Introduction and statement of results}

The concept of Poissonian pair correlations has its origin in quantum mechanics, where the spacings of energy levels of integrable systems were studied. See for example [1] and the references cited therein for detailed information on that topic. Rudnick and Sarnak first studied this concept from a purely mathematical point of view and over the years the topic has attracted wide attention, see e.g., [12,17-20].

Let $\|\cdot\|$ denote the distance to the nearest integer. A sequence $\left(x_{n}\right)_{n \in \mathbb{N}}$ of real numbers in $[0,1)$ has Poissonian pair correlations if the pair correlation statistics

$$
F_{N}(s):=\frac{1}{N} \#\left\{1 \leq l \neq m \leq N:\left\|x_{l}-x_{m}\right\| \leq \frac{s}{N}\right\}
$$

tends to $2 s$, for every $s \geq 0$, as $N \rightarrow \infty$.

Let now $d \geq 2$ be an integer denoting the dimension of the problem setting. In the sequel, we indicate by bold symbols that we work with $d$-dimensional vectors of real numbers or random variables. We extend the above notion to sequences $\left(\boldsymbol{x}_{n}\right)_{n \in \mathbb{N}}$ in the $d$-dimensional unit cube $[0,1)^{d}$. Subsequently, we denote by $\|\cdot\|_{\infty}$ a supremum-norm of a $d$-dimensional vector, i.e., in our case for some $\boldsymbol{x}=\left(x_{1}, \ldots, x_{d}\right) \in \mathbb{R}^{d}$,

$$
\|x\|_{\infty}:=\max \left(\left\|x_{1}\right\|, \ldots,\left\|x_{d}\right\|\right)
$$

where we recall that $\|\cdot\|$ denotes the distance to the nearest integer.

We say that a sequence $\left(\boldsymbol{x}_{n}\right)_{n \in \mathbb{N}} \in[0,1)^{d}$ has Poissonian pair correlations if the multi-dimensional pair correlation statistics

$$
F_{N}^{(d)}(s):=\frac{1}{N} \#\left\{1 \leq l \neq m \leq N:\left\|\boldsymbol{x}_{l}-\boldsymbol{x}_{m}\right\|_{\infty} \leq \frac{s}{N^{1 / d}}\right\}
$$

tends to $(2 s)^{d}$, for every $s \geq 0$, as $N \rightarrow \infty$.

In the one-dimensional case, it is well-known that for a sequence of i.i.d. random variables $\left(X_{i}\right)_{i \in \mathbb{N}}$ having uniform distribution on $[0,1), F_{N}(s)$ tends to $2 s$, as $N \rightarrow \infty$, almost surely (see e.g., $[2,16])$. Clearly, a multi-dimensional analogue of this statement holds as well.

Proposition 1 Let $\left(\boldsymbol{X}_{i}\right)_{i \in \mathbb{N}}$ a sequence of i.i.d. random variables having uniform distribution on $[0,1)^{d}$, then for all $s>0$, we have $F_{N}^{(d)}(s) \rightarrow(2 s)^{d}$, as $N \rightarrow \infty$, almost surely.

The proof is based on the pairwise independence of the random variables $\boldsymbol{X}_{l}-\boldsymbol{X}_{m}$, $l \neq m$, and the Borel-Cantelli lemma. For completeness, we state the proof in Sect. 2. Note that, however, this result would be true if the " $\ell_{\infty}$-ball" in the definition of the pair correlation is replaced by an arbitrary measurable set of volume $(2 s)^{d} / N$.

The notion of Poissonian pair correlation has attracted renewed interest in the last few years, due to its connection to several mathematical fields, such as Diophantine approximation, additive combinatorics and uniform distribution (see e.g., [1,3,4,12,14, 22]). The link between the concept of uniform distribution modulo 1 and Poissonian 
pair correlation has been studied in the one-dimensional case. Due to a result by Grepstad and Larcher [11] (see also [2,21]), we know that a sequence which satisfies that (1) tends to $2 s$, for every $s>0$, as $N \rightarrow \infty$, is also uniformly distributed in $[0,1)$, i.e., it satisfies

$$
\lim _{N \rightarrow \infty} \frac{1}{N} \#\left\{1 \leq n \leq N: x_{n} \in[a, b)\right\}=b-a
$$

for all $0 \leq a<b \leq 1$. The above presented multi-dimensional concept of Poissonian pair correlation also implies uniform distribution of a sequence in $[0,1)^{d}$, i.e.,

$$
\lim _{N \rightarrow \infty} \frac{1}{N} \#\left\{1 \leq n \leq N: \boldsymbol{x}_{n} \in[\boldsymbol{a}, \boldsymbol{b})\right\}=\prod_{i=1}^{d}\left(b_{i}-a_{i}\right),
$$

for all $\mathbf{0} \leq \boldsymbol{a}<\boldsymbol{b} \leq \mathbf{1}$, where $\boldsymbol{a}<\boldsymbol{b}$ has to be understood componentwise, i.e., $a_{i}<b_{i}$ for $1 \leq i \leq d$.

Theorem 1 Let $\left(\boldsymbol{x}_{n}\right)_{n \in \mathbb{N}} \in[0,1)^{d}$ be such that for every $s \in \mathbb{N}$ we have that

$$
\lim _{N \rightarrow \infty} F_{N}^{(d)}(s)=(2 s)^{d},
$$

then $\left(\boldsymbol{x}_{n}\right)_{n \in \mathbb{N}}$ is uniformly distributed in $[0,1)^{d}$

It turns out by the proof of this theorem that it is sufficient to have the Poissonian property for positive integer-valued $s$ only in order to deduce uniform distribution for a sequence $\left(\boldsymbol{x}_{n}\right)_{n \in \mathbb{N}} \in[0,1)^{d}$ (the same holds in the one-dimensional case as well). It would be very interesting if this result also holds if we define the multidimensional pair correlation concept using other norms, e.g., the $L_{2}$-norm. However, it is not straightforward how one could prove such a result. The main difficulty here is the following: we can pave the unit-cube with a suitable choice of boxes of volume $1 / N$ when we work with the supremum-norm. Such a partitioning is not possible, when using the $L_{2}$-norm.

This new concept of course raises several further questions. E.g., in the onedimensional case, it is known that for almost all choices of $\alpha$ the sequence $(\{f(n) \alpha\})_{n \in \mathbb{N}}$, where $f(x)$ is a polynomial of degree at least 2 with integer coefficients, has Poissonian pair correlations [18]. If a $d$-dimensional polynomial $\boldsymbol{p}(x)=$ $\left(p_{1}(x), \ldots, p_{d}(x)\right)\left(p_{i}(x)\right.$ are all real polynomials) has the property that for each lattice point $\boldsymbol{h} \in \mathbb{Z}^{d}, \boldsymbol{h} \neq \mathbf{0}$ the polynomial $\langle\boldsymbol{h}, \boldsymbol{p}(x)\rangle$ has at least one non-constant term with irrational coefficient, then

$$
\left(\left(\left\{p_{1}(n)\right\}, \ldots,\left\{p_{d}(n)\right\}\right)\right)_{n \in \mathbb{N}}
$$

is uniformly distributed in $[0,1)^{d}$ (see e.g., [13]). Therefore, in analogy to the onedimensional case, it would be natural to expect that for an integer polynomial $f(x)$ with degree at least 2 , the sequence

$$
\left(\left(\left\{f(n) \alpha_{1}\right\}, \ldots,\left\{f(n) \alpha_{d}\right\}\right)\right)_{n \in \mathbb{N}},
$$


has Poissonian pair correlation for almost all choices of $\alpha_{1}, \ldots, \alpha_{d}$ (this claim will be a consequence of Theorem 2).

To be more general, let $\left(a_{n}\right)_{n \in \mathbb{N}}$ be an increasing sequence of distinct integers and $\boldsymbol{\alpha}=\left(\alpha_{1}, \ldots, \alpha_{d}\right)$. Then we consider a sequence of the form

$$
\left(\left\{a_{n} \boldsymbol{\alpha}\right\}\right)_{n \in \mathbb{N}}:=\left(\left(\left\{a_{n} \alpha_{1}\right\}, \ldots,\left\{a_{n} \alpha_{d}\right\}\right)\right)_{n \in \mathbb{N}}
$$

which is uniformly distributed for almost all choices of $\alpha_{1}, \ldots, \alpha_{d}$.

In the one-dimensional case the metrical pair correlation theory of such sequences is strongly linked to the additive energy of a finite integer set $A$, denoted by $E(A)$. The additive energy $E(A)$ is defined as

$$
E(A):=\sum_{a+b=c+d} 1,
$$

where the sum is extended over all quadruples $(a, b, c, d) \in A^{4}$. This connection was discovered by Aistleitner, Larcher and Lewko, who, roughly speaking, proved in [4] that if the first $N$ elements of an increasing sequence of distinct integers $\left(a_{n}\right)_{n \in \mathbb{N}}$, have an arbitrarily small energy saving, then $\left(\left\{a_{n} \alpha\right\}\right)_{n \in \mathbb{N}}$ has Poissonian pair correlations for almost all $\alpha$. Recently, Bloom and Walker (see [5]) improved over this result by showing the following theorem.

Theorem A There exists an absolute positive constant $C$ such that the following is true. Let $A_{N}$ denote the first $N$ elements of $\left(a_{n}\right)_{n \in \mathbb{N}}$ and suppose that

$$
E\left(A_{N}\right)=\mathcal{O}\left(\frac{N^{3}}{(\log N)^{C}}\right),
$$

then for almost all $\alpha,\left(\left\{a_{n} \alpha\right\}\right)_{n \in \mathbb{N}}$ has Poissonian pair correlations.

The proof of this result relies on a new bound for GCD sums with exponent $1 / 2$, which improves over the bound by Bondarenko and Seip (see [6]), if the additive energy of $A_{N}$ is sufficiently large. Note that the constant $C$ was not specified in the above mentioned paper, but the authors thereof conjecture that Theorem A holds for $C>1$ already. This result would be best possible. To see this, consider the sequence $\left(p_{n}\right)_{n \in \mathbb{N}}$ of all primes. It is known that $\left(p_{n}\right)_{n=1}^{N}$ has additive energy of exact order $N^{3} /(\log N)$, but $\left(\left\{p_{n} \alpha\right\}\right)_{n \in \mathbb{N}}$ is not metric Poissonian, i.e., there exists a set $\Omega$ of full Lebesgue measure, such that for all $\alpha \in \Omega,\left(\left\{p_{n} \alpha\right\}\right)_{n \in \mathbb{N}}$ does not have Poissonian pair correlations (see [22]).

Naturally, we would also expect that under this condition on the additive energy, the sequence

$$
\left(\left\{a_{n} \boldsymbol{\alpha}\right\}\right)_{n \in \mathbb{N}}
$$

has Poissonian pair correlations for almost all instances and, in fact, we have the following even better result, which is a consequence of better bounds on GCD sums for larger exponents than $1 / 2$ : 
Theorem 2 Let $A_{N}$ denote the first $N$ elements of $\left(a_{n}\right)_{n \in \mathbb{N}}$ and suppose that

$$
E\left(A_{N}\right)=\mathcal{O}\left(\frac{N^{3}}{(\log N)^{1+\varepsilon}}\right), \text { for any } \varepsilon>0,
$$

then for almost all choices of $\boldsymbol{\alpha}=\left(\alpha_{1}, \ldots, \alpha_{d}\right) \in \mathbb{R}^{d}$,

$$
\left(\left\{a_{n} \boldsymbol{\alpha}\right\}\right)_{n \in \mathbb{N}}
$$

has Poissonian pair correlations.

However, if the additive energy is of maximal order, i.e., if we have $E\left(A_{N}\right)=\Omega\left(N^{3}\right)$, then there is no $\alpha$ such that $\left(\left\{a_{n} \alpha\right\}\right)_{n \in \mathbb{N}}$ has Poissonian pair correlations, see [14]. The approach used in [14] can be generalised to arbitrary dimensions.

Theorem 3 If $E\left(A_{N}\right)=\Omega\left(N^{3}\right)$, then for any choice of $\boldsymbol{\alpha}=\left(\alpha_{1}, \ldots, \alpha_{d}\right) \in \mathbb{R}^{d}$ the sequence

$$
\left(\left\{a_{n} \boldsymbol{\alpha}\right\}\right)_{n \in \mathbb{N}},
$$

does not have Poissonian pair correlations.

Classical low-discrepancy sequences in $[0,1)$, e.g., the van der Corput sequence, the Kronecker sequence $(\{n \alpha\})_{n \in \mathbb{N}}$ and digital $(t, 1)$-sequences in base $b \geq 2$, do not have Poissonian pair correlations (see e.g., [15]). We will derive an analogous result for the multi-dimensional version of the Kronecker sequence.

Corollary 1 For any choice of $\boldsymbol{\alpha}=\left(\alpha_{1}, \ldots, \alpha_{d}\right) \in \mathbb{R}^{d}$ the d-dimensional Kronecker sequence

$$
\left(\boldsymbol{x}_{n}\right)_{n \in \mathbb{N}}:=(\{n \boldsymbol{\alpha}\})_{n \in \mathbb{N}},
$$

where $\{\cdot\}$ denotes the fractional part of a real number, does not have Poissonian pair correlations.

Of course, Corollary 1 is an immediate consequence of Theorem 3. However, we also include an explicit proof of Corollary 1 as an "Appendix", since it gives an intuitive feeling for the multi-dimensional Poissonian pair correlation concept.

We also strongly believe that other multi-dimensional low-discrepancy sequences such as $(t, s)$-sequences and the Halton sequence do not have Poissonian pair correlations.

Remark 1 Although the metrical theory of sequences of the form $\left(\left\{a_{n} \boldsymbol{\alpha}\right\}\right)_{n \in \mathbb{N}}$ seems to be well-established, we do not know any explicit construction of $\boldsymbol{\alpha}$ (not even in the one-dimensional case) such that $\left(\left\{a_{n} \boldsymbol{\alpha}\right\}\right)_{n \in \mathbb{N}}$ has Poissonian pair correlations. It is in general very hard to construct sequences having Poissonian pair correlations. The only known explicit examples — to the best of our knowledge — of sequences with this property are $\{\sqrt{n}\}_{n \in \mathbb{N}}$ (see [8]) and certain directions of vectors in an affine Euclidean lattice (see [9]). 


\section{Proof of Proposition 1}

As $\left(\boldsymbol{X}_{i}\right)_{i \in \mathbb{N}}$ is a sequence of i.i.d. random variables having uniform distribution on $[0,1)^{d}$, we have

$$
\begin{aligned}
\mathbb{E}\left(F_{N}^{(d)}(s)\right) & =\mathbb{E}\left(\frac{1}{N} \#\left\{1 \leq k \neq l \leq N:\left\|\boldsymbol{X}_{k}-\boldsymbol{X}_{l}\right\|_{\infty} \leq \frac{s}{N^{1 / d}}\right\}\right) \\
& =\frac{1}{N} N(N-1) \int_{[0,1)^{d}} \int_{x_{1}-s / N^{1 / d}}^{x_{1}+s / N^{1 / d}} \cdots \int_{x_{d}-s / N^{1 / d}}^{x_{d}+s / N^{1 / d}} 1 d \boldsymbol{y} d x_{d} \ldots d x_{1} \\
& =\frac{N-1}{N}(2 s)^{d} .
\end{aligned}
$$

Due to the fact that the random variables $\boldsymbol{X}_{k}-\boldsymbol{X}_{l}, k \neq l$, (note that the difference is considered modulo 1) are pairwise independent, we obtain that the variance of $F_{N}^{(d)}(s)$, denoted by $\sigma^{2}\left(F_{N}^{(d)}(s)\right)$, satisfies, whenever $N \geq(2 s)^{d}$,

$$
\begin{aligned}
\sigma^{2}\left(F_{N}^{(d)}(s)\right) & =\frac{1}{N^{2}} \sum_{1 \leq k \neq l \leq N} \sigma^{2}\left(\mathbf{1}\left(\left\|\boldsymbol{X}_{k}-\boldsymbol{X}_{l}\right\|_{\infty} \leq \frac{s}{N^{1 / d}}\right)\right) \\
& =\frac{N(N-1)}{N^{2}} \frac{(2 s)^{d}}{N}\left(1-\frac{(2 s)^{d}}{N}\right) \\
& \leq \frac{(2 s)^{d}}{N}
\end{aligned}
$$

where 1(.) denotes the indicator function. Using Chebyshev's inequality, we obtain that for all $\epsilon, s>0$,

$$
\mathbb{P}\left(\left|F_{N}^{(d)}(s)-\mathbb{E}\left(F_{N}^{(d)}(s)\right)\right| \geq \epsilon\right) \leq \frac{(2 s)^{d}}{\epsilon^{2} N},
$$

if $N$ is large enough. To prove now almost sure convergence, one can apply the arguments used in [4,20]. We fix a $\gamma>0$ and define a subsequence $N_{M}$ along the integers, for $M \geq 1$, as

$$
N_{M}:=M^{1+\gamma}
$$

The variance estimate from above combined with Chebyshev's inequality and the first Borel-Cantelli lemma allow to deduce that, for all $s>0$,

$$
\lim _{M \rightarrow \infty} F_{N_{M}}^{(d)}(s)=(2 s)^{d}, \text { almost surely, }
$$

i.e., we have now almost sure convergence along a subsequence of the integers. For $N$, with $N_{M} \leq N \leq N_{M+1}$, we use the trivial bounds 


$$
\begin{aligned}
N_{M} F_{N_{M}}^{(d)}\left(\frac{N_{M}}{N_{M+1}} s\right) & \leq N F_{N}^{(d)}(s) \\
& \leq N_{M+1} F_{N_{M+1}}^{(d)}\left(\frac{N_{M+1}}{N_{M}} s\right) .
\end{aligned}
$$

Since $N_{M+1} / N_{M} \rightarrow 1$, as $M \rightarrow \infty$, we also get

$$
\lim _{N \rightarrow \infty} F_{N}^{(d)}(s)=(2 s)^{d} \text {, almost surely. }
$$

\section{Proof of Theorem 1}

We carry out the proof for $d=2$, for an arbitrary $d$ the arguments run quite analogously. To prove the theorem, we assume in the contrary that $\left(\boldsymbol{x}_{n}\right)_{n \in \mathbb{N}}$ is not uniformly distributed and will derive a contradiction. Due to this assumption, there exists an $\epsilon>0$ and $\alpha, \beta$ with $0<\alpha, \beta<1$ such that

$$
\left|\frac{1}{N} \#\left\{1 \leq n \leq N: \boldsymbol{x}_{n} \in[0, \alpha) \times[0, \beta)\right\}-\alpha \beta\right|>\epsilon,
$$

for infinitely many $N$. Note that for simplicity, we work here with boxes anchored at the origin. One can readily adopt the following steps to arbitrary axis-parallel boxes. Hence, we can assume that for an increasing sequence of integers $\left(N_{i}\right)_{i \in \mathbb{N}}$ we have

$$
\frac{1}{N_{i}} \#\left\{1 \leq n \leq N_{i}: \boldsymbol{x}_{n} \in[0, \alpha) \times[0, \beta)\right\} \leq \alpha \beta-\epsilon
$$

(The case that we have " $\geq \alpha \beta+\epsilon$ " in the above expression can be treated analogously.) Let $N:=N_{i}$ for some $i \geq 1$ and assume for simplicity that $\sqrt{N}$ is an integer. For $0 \leq i, j<\sqrt{N}$ let

$$
A_{i, j}:=\#\left\{1 \leq n \leq N: \boldsymbol{x}_{n} \in\left[\frac{i}{\sqrt{N}}, \frac{i+1}{\sqrt{N}}\right) \times\left[\frac{j}{\sqrt{N}}, \frac{j+1}{\sqrt{N}}\right)\right\}
$$

If $i$ and/or $j \geq \sqrt{N}$, we set

$$
A_{i, j}:=A_{i \bmod \sqrt{N}, j \bmod \sqrt{N}} .
$$


Then for all integers $s$ and $N$ large enough, we have

$$
\begin{aligned}
N F_{N}^{(d)}(s) & \geq \sum_{i, j=0}^{\sqrt{N}-1} A_{i, j}\left(A_{i, j}-1\right)+2 A_{i, j} B_{i, j}, \text { where } \\
B_{i, j} & :=\sum_{u=1}^{s-1} \sum_{v=-(s-1)}^{s-1} A_{i+u, j+v}+\sum_{v=1}^{s-1} A_{i, j+v} .
\end{aligned}
$$

Hence,

$$
N F_{N}^{(d)}(s) \geq \sum_{i, j=0}^{\sqrt{N}-1}\left(\left(A_{i, j}+B_{i, j}\right)^{2}-B_{i, j}^{2}\right)-N
$$

as we have

$$
\sum_{i, j=0}^{\sqrt{N}-1} A_{i, j}=N
$$

If $\alpha=\frac{a}{\sqrt{N}}$ and $\beta=\frac{b}{\sqrt{N}}$ (assume for simplicity that $a$ and $b$ are positive integers), then we have

$$
\sum_{i=0}^{a-1} \sum_{j=0}^{b-1} A_{i, j} \leq N(\alpha \beta-\epsilon)
$$

and

$$
\sum_{\substack{i, j \\ i \geq a \text { or } j \geq b}} A_{i, j} \geq N(1-\alpha \beta+\epsilon)
$$

Now, considering a standard constrained optimization problem, we get in a first step that the quadratic form

$$
\sum_{i, j=0}^{\sqrt{N}-1}\left(\left(A_{i, j}+B_{i, j}\right)^{2}-B_{i, j}^{2}\right)
$$

attains its minimum under conditions (2), (3) and (4) if in (3) and (4) we have equality. In a second step, it can be shown that (5) attains its minimum under conditions (2), (3) and (4) (with equality sign) if all $A_{i, j}$ occurring in the sum of (3) and (4) have the same value. This means the minimum is attained if

$$
A_{i, j}=\left\{\begin{array}{l}
\frac{N(\alpha \beta-\epsilon)}{a b}=1-\frac{\epsilon}{\alpha \beta}, \quad \text { if } 0 \leq i<a \text { and } 0 \leq j<b, \\
\frac{N(1-\alpha \beta+\epsilon)}{N-a b}=1+\frac{\epsilon}{1-\alpha \beta}, \quad \text { otherwise. }
\end{array}\right.
$$


Note that each $B_{i, j}$ consists of $\frac{(2 s-1)^{2}-1}{2}=2 s(s-1)$ summands $A_{x, y}$ and therefore,

$$
\begin{aligned}
N F_{N}^{(d)}(s) \geq & \sum_{i=0}^{a-1} \sum_{j=0}^{b-1}\left(\left(A_{i, j}+B_{i, j}\right)^{2}-B_{i, j}^{2}\right) \\
& +\sum_{\substack{i, j \\
i \geq a \text { or } j \geq b}}\left(\left(A_{i, j}+B_{i, j}\right)^{2}-B_{i, j}^{2}\right)-N \\
\geq & a b\left(\left((2 s(s-1)+1)\left(1-\frac{\epsilon}{\alpha \beta}\right)\right)^{2}-\left((2 s(s-1))\left(1-\frac{\epsilon}{\alpha \beta}\right)\right)^{2}\right) \\
& +(N-a b)\left(\left((2 s(s-1)+1)\left(1+\frac{\epsilon}{1-\alpha \beta}\right)\right)^{2}\right. \\
& \left.-\left((2 s(s-1))\left(1+\frac{\epsilon}{1-\alpha \beta}\right)\right)^{2}\right)-N \\
= & (4 s(s-1)+1)\left[a b\left(1-\frac{\epsilon}{\alpha \beta}\right)^{2}+(N-a b)\left(1+\frac{\epsilon}{1-\alpha \beta}\right)^{2}\right]-N \\
= & N\left[(4 s(s-1)+1)\left(\lambda\left(1-\frac{\epsilon}{\lambda}\right)^{2}+(1-\lambda)\left(1+\frac{\epsilon}{1-\lambda}\right)^{2}\right)^{2}\right] \\
= & N R_{\epsilon, \lambda}(s),
\end{aligned}
$$

where $\lambda:=\alpha \beta$. By assumption, we have $\lim _{N \rightarrow \infty} F_{N}^{(d)}(s)=(2 s)^{2}$ for all positive integers $s$. Therefore, in order to derive a contradiction, it suffices to show that there exists an integer $s$ such that

$$
R_{\epsilon, \lambda}(s)>(2 s)^{2} .
$$

The expression $R_{\epsilon, \lambda}(s)-(2 s)^{2}$ can be viewed as a quadratic polynomial in $s$ with leading coefficient

$$
4 \lambda\left(1-\frac{\epsilon}{\lambda}\right)^{2}+4(1-\lambda)\left(1+\frac{\epsilon}{1-\lambda}\right)^{2}-4=4 \frac{\epsilon^{2}}{\lambda(1-\lambda)}>0 .
$$

Hence (6) holds for all $s$ large enough in dependence on $\epsilon$ and $\lambda$.

\section{Proof of Theorem 2}

We adapt the steps of Lemma 3 of [4], which we will shortly repeat here.

In the sequel let $d \geq 2$ and $1, \alpha_{1}, \ldots, \alpha_{d}$ be linearly independent over the rationals. For $\boldsymbol{\alpha}=\left(\alpha_{1}, \ldots, \alpha_{d}\right)$, we denote by $\boldsymbol{I}_{N, s}(\boldsymbol{\alpha})$, for a fixed $s \asymp 1$ and $N$, with $(2 s)^{d} \leq N$, the indicator function of the interval 


$$
\left[-s / N^{1 / d}, s / N^{1 / d}\right)^{d}
$$

extended with period 1 . Therefore, we can write the pair correlation function $F_{N}^{(d)}(s)$ (subsequently interpreted as a function of $\boldsymbol{\alpha})$ for the sequence $\left(\left\{a_{n} \boldsymbol{\alpha}\right\}\right)_{n \in \mathbb{N}}$ as

$$
F_{N, s}^{(d)}(\boldsymbol{\alpha}):=\frac{1}{N} \sum_{\substack{1 \leq k, l \leq N \\ k \neq l}} \boldsymbol{I}_{N, s}\left(\boldsymbol{\alpha}\left(a_{k}-a_{l}\right)\right)
$$

We consider the Fourier series expansion of $\boldsymbol{I}_{N, s}(\boldsymbol{\alpha})$, i.e.,

$$
\boldsymbol{I}_{N, S}(\boldsymbol{\alpha}) \sim \sum_{\boldsymbol{r} \in \mathbb{Z}^{d}} c_{\boldsymbol{r}} e(\langle\boldsymbol{r}, \boldsymbol{\alpha}\rangle)
$$

with

$$
c_{\boldsymbol{r}}=\int_{-s / N^{1 / d}}^{s / N^{1 / d}} \cdots \int_{-s / N^{1 / d}}^{s / N^{1 / d}} e^{-2 \pi i \sum_{i=1}^{d} r_{i} \alpha_{i}} d \alpha_{1} \ldots d \alpha_{d}
$$

where $\boldsymbol{r}=\left(r_{1}, \ldots, r_{d}\right)$. Hence, we can write $c_{\boldsymbol{r}}=c_{r_{1}} \ldots c_{r_{d}}$, where $c_{r_{j}}=$ $\int_{-s / N^{1 / d}}^{s / N^{1 / d}} e^{-2 \pi i r_{j} \alpha_{j}} d \alpha_{j}$, for $j=1, \ldots, d$. Note that we have

$$
\left|c_{r_{j}}\right| \leq \min \left(\frac{2 s}{N^{1 / d}}, \frac{1}{\left|r_{j}\right|}\right)
$$

This gives the following expression for the variance of $F_{N, s}^{(d)}(\boldsymbol{\alpha})$. Note, due to the assumption on $s$, the constants implied by "«" are independent of $s$ (and of course independent of $N$ ).

$$
\begin{aligned}
& \int_{[0,1)^{d}}\left(F_{N, s}^{(d)}(\boldsymbol{\alpha})-\frac{(2 s)^{d}(N-1)}{N}\right)^{2} d \boldsymbol{\alpha} \\
& =\frac{1}{N^{2}} \int_{[0,1)^{d}}\left(\sum_{\substack{1 \leq k, l \leq N \\
k \neq l}} \sum_{\boldsymbol{r} \in \mathbb{Z}^{d} \backslash\{\boldsymbol{0}\}} c_{\boldsymbol{r}} e\left(\left\langle\boldsymbol{r}, \boldsymbol{\alpha}\left(a_{k}-a_{l}\right)\right\rangle\right)\right)^{2} d \boldsymbol{\alpha} \\
& =\frac{1}{N^{2}} \sum_{\substack{1 \leq k, l, m, n \leq N \\
k \neq l, m \neq n}} \sum_{\boldsymbol{r}_{1}, \boldsymbol{r}_{2} \in \mathbb{Z}^{d} \backslash\{\mathbf{0}\}}\left|c_{\boldsymbol{r}_{1}} c_{\boldsymbol{r}_{2}}\right| \times \\
& \times \int_{[0,1)^{d}} e\left(\left\langle\boldsymbol{r}_{1}, \boldsymbol{\alpha}\left(a_{k}-a_{l}\right)\right\rangle-\left\langle\boldsymbol{r}_{2}, \boldsymbol{\alpha}\left(a_{m}-a_{n}\right)\right\rangle\right) d \boldsymbol{\alpha} \\
& =\frac{1}{N^{2}} \sum_{\substack{1 \leq k, l, m, n \leq N \\
k \neq l, m \neq n}} \sum_{\boldsymbol{r}_{1}, \boldsymbol{r}_{2} \in \mathbb{Z}^{d} \backslash\{\mathbf{0}\}}\left|c_{\boldsymbol{r}_{1}} c_{\boldsymbol{r}_{2}}\right| \int_{[0,1)}
\end{aligned}
$$




$$
\begin{aligned}
& e\left(\alpha_{1}\left(r_{1}^{(1)}\left(a_{k}-a_{l}\right)-r_{2}^{(1)}\left(a_{m}-a_{n}\right)\right)\right) d \alpha_{1} \ldots \\
& \times \int_{[0,1)} e\left(\alpha_{d}\left(r_{1}^{(d)}\left(a_{k}-a_{l}\right)-r_{2}^{(d)}\left(a_{m}-a_{n}\right)\right)\right) d \alpha_{d}
\end{aligned}
$$

with $\boldsymbol{r}_{i}=\left(r_{i}^{(1)}, \ldots, r_{i}^{(d)}\right)$ for $i=1,2$.

Recalling that the representation function $r_{N}(v)$ of an integer $v$ is defined as

$$
r_{N}(v):=\#\left\{1 \leq k \neq l \leq N: a_{k}-a_{l}=v\right\},
$$

we can write the previous expression as

$$
\frac{1}{N^{2}} \sum_{v, w \in \mathbb{Z} \backslash\{0\}} r_{N}(v) r_{N}(w) \sum_{\substack{\boldsymbol{r}_{1}, \boldsymbol{r}_{2} \in \mathbb{Z}^{d} \backslash\{\mathbf{0}\} \\ r_{1}^{(i)} v=r_{2}^{(i)} w}}^{\prime}\left|c_{\boldsymbol{r}_{1}} c_{\boldsymbol{r}_{2}}\right|,
$$

where in the sum $\sum^{\prime}$ the equality $r_{1}^{(i)} v=r_{2}^{(i)} w$ only needs to hold for these indices $i$ for which $r_{1}^{(i)}, r_{2}^{(i)} \neq 0$. To further estimate the expression (8), we first derive the estimate

$$
\sum_{\substack{\boldsymbol{r}_{1}, \boldsymbol{r}_{2} \in \mathbb{Z}^{d} \backslash\{\mathbf{0}\} \\ r_{1}^{(i)} v=r_{2}^{(i)} w}}\left|c_{\boldsymbol{r}_{1}} c_{\boldsymbol{r}_{2}}\right| \ll \frac{s^{d} \log N}{N} \frac{\operatorname{gcd}(v, w)^{d}}{\sqrt{|v w|^{d}}}, \quad v, w \neq 0,
$$

where the implied constant depends on $d$ and all entries of $\boldsymbol{r}_{1}$ and $\boldsymbol{r}_{2}$ are assumed to be non-zero. Note that compared to the one-dimensional case the exponent appearing in the GCD expression is now $\geq 2$, which allows sharper estimates of the corresponding GCD sum. To see that (9) is true, we recall that $r_{1}^{(i)} v=r_{2}^{(i)} w$ for $i=1, \ldots, d$ if and only if

$$
r_{1}^{(i)}=\frac{h_{i} w}{\operatorname{gcd}(v, w)}, \text { and } r_{2}^{(i)}=\frac{h_{i} v}{\operatorname{gcd}(v, w)} \text { for } i=1, \ldots, d \text {, }
$$

where the $h_{i}$ 's are some integers. Then, case distinctions according to the size of the $h_{i}$ 's, i.e.,

$$
\begin{aligned}
& \left|h_{i}\right| \leq \frac{N^{1 / d} \operatorname{gcd}(v, w)}{s \max (|v|,|w|)}=: \max _{h_{i}} \\
& \frac{N^{1 / d} \operatorname{gcd}(v, w)}{s \max (|v|,|w|)} \leq\left|h_{i}\right| \leq \frac{N^{1 / d} \operatorname{gcd}(v, w)}{s \min (|v|,|w|)}=: \min _{h_{i}} \\
& \left|h_{i}\right| \geq \frac{N^{1 / d} \operatorname{gcd}(v, w)}{s \min (|v|,|w|)}
\end{aligned}
$$


and recalling the bounds on the Fourier coefficients (7) gives the following estimate. For fixed $v, w$, and $\mathcal{D}:=\{1, \ldots, d\}$, we have

$$
\begin{aligned}
& \sum_{\substack{\boldsymbol{r}_{1}, \boldsymbol{r}_{2} \in \mathbb{Z}^{d} \backslash\{\mathbf{0}\} \\
r_{1}^{(i)} v=r_{2}^{(i)} w}}\left|c_{\boldsymbol{r}_{1}} c_{\boldsymbol{r}_{2}}\right|=\sum_{\substack{\boldsymbol{r}_{1}, \boldsymbol{r}_{2} \in \mathbb{Z}^{d} \backslash\{\mathbf{0}\} \\
r_{1}^{(i)} v=r_{2}^{(i)} w}}\left|c_{r_{1}^{(1)}} \ldots c_{r_{1}^{(d)}} c_{r_{2}^{(1)}} \ldots c_{r_{2}^{(d)}}\right|
\end{aligned}
$$

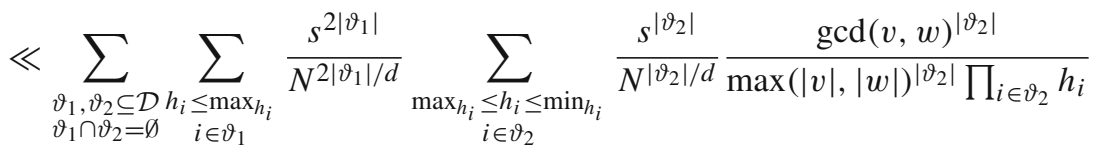

$$
\begin{aligned}
& \times \sum_{\substack{h_{i} \geq \min _{h_{i}} \\
i \in \mathcal{D} \backslash\left(\vartheta_{1} \cup \vartheta_{2}\right)}} \frac{\operatorname{gcd}(v, w)^{2\left(d-\left|\vartheta_{1} \cup \vartheta_{2}\right|\right)}}{|v w|^{d-\left|\vartheta_{1} \cup \vartheta_{2}\right|} \prod_{i \in \mathcal{D} \backslash\left(\vartheta_{1} \cup \vartheta_{2}\right)} h_{i}^{2}} \\
& \ll \sum_{\substack{\vartheta_{1}, \vartheta_{2} \subseteq \mathcal{D} \\
\vartheta_{1} \cap \vartheta_{2}=\emptyset}} \frac{N^{\left|\vartheta_{1}\right| / d} \operatorname{gcd}(v, w)^{\left|\vartheta_{1}\right|}}{s^{\left|\vartheta_{1}\right| \max (|v|,|w|)^{\left|\vartheta_{1}\right|}}} \frac{s^{2|\vartheta|_{1}}}{N^{2\left|\vartheta_{1}\right| / d}} \times \\
& \times \log N \frac{s^{\left|\vartheta_{2}\right|}}{N^{\left|\vartheta_{2}\right| / d}} \frac{\operatorname{gcd}(v, w)^{\left|\vartheta_{2}\right|}}{\max (|v|,|w|)^{\left|\vartheta_{2}\right|}} \frac{\operatorname{gcd}(v, w)^{2\left(d-\left|\vartheta_{1} \cup \vartheta_{2}\right|\right)}}{|v w|^{d-\left|\vartheta_{1} \cup \vartheta_{2}\right|}} \\
& \times \frac{s^{\left(d-\left|\vartheta_{1} \cup \vartheta_{2}\right|\right)}}{N^{\left(d-\left|\vartheta_{1} \cup \vartheta_{2}\right|\right) / d}} \frac{\min (|v|,|w|)^{\left(d-\left|\vartheta_{1} \cup \vartheta_{2}\right|\right)}}{\operatorname{gcd}(v, w)^{\left(d-\left|\vartheta_{1} \cup \vartheta_{2}\right|\right)}} \\
& \ll \frac{s^{d} \log N}{N} \frac{\operatorname{gcd}(v, w)^{d}}{\sqrt{|v w|^{d}}} \text {. }
\end{aligned}
$$

Consequently, we have for the variance of $F_{N, s}^{(d)}(\boldsymbol{\alpha})$, using the result on GCD sums by Gál (see [10]),

$$
\begin{aligned}
& \int_{[0,1)^{d}}\left(F_{N, s}^{(d)}(\boldsymbol{\alpha})-\frac{(2 s)^{d}(N-1)}{N}\right)^{2} d \boldsymbol{\alpha} \\
& =\frac{1}{N^{2}} \sum_{v, w \in \mathbb{Z} \backslash\{0\}} r_{N}(v) r_{N}(w) \sum_{\substack{\boldsymbol{r}_{1}, \boldsymbol{r}_{2} \in \mathbb{Z}^{d} \backslash\{\boldsymbol{0}\} \\
r_{1}^{(i)} v=r_{2}^{(i)} w}}^{\prime}\left|c_{\boldsymbol{r}_{1}} c_{\boldsymbol{r}_{2}}\right| \\
& \ll \frac{s^{d} \log N}{N^{3}} \sum_{v, w \in \mathbb{Z} \backslash\{0\}} r_{N}(v) r_{N}(w) \frac{\operatorname{gcd}(v, w)^{d}}{\sqrt{|v w|^{d}}} \\
& \ll\left\{\begin{array}{l}
\frac{s^{d} E\left(A_{N}\right) \log N}{N^{3}}(\log \log N)^{2}, \text { for } d=2, \\
\frac{s^{d} E\left(A_{N}\right) \log N}{N^{3}}, \text { for } d \geq 3 .
\end{array}\right.
\end{aligned}
$$

Then, using similar arguments as for example in the proof of Theorem 6 of [5], we can deduce the claim. 


\section{Proof of Theorem 3}

The proof uses exactly the same steps, except some minor technical changes, as the one in the one-dimensional case, see [14]. To make this paper self-contained, we repeat the most important steps here. We demonstrate the proof for $d=2$ only, as for general $d$ the arguments are quite analogue. As $\left(a_{n}\right)_{n \in \mathbb{N}}$ has maximal order of additive energy it is well-known (see [1]) that there exist constants $C, K>0$ and a strictly increasing sequence of positive integers $\left(N_{i}\right)_{i}$ such that for all $i \geq 1$ there is a subset $A^{(i)} \subset\left(a_{n}\right)_{1 \leq n \leq N_{i}}$ with $\left|A^{(i)}\right| \geq C N_{i}$ such that $A^{(i)}$ is contained in a $q$-dimensional arithmetic progression $P_{i}$ (for some integer $q$ ) of size at most $K N_{i}$. We call such an integer sequence quasi-arithmetic of degree $q$.

To be more precise, we have the following: For all $i \geq 1$ there is a subset $b_{1}<b_{2}<\cdots<b_{M_{i}}$ of $\left(a_{n}\right)_{n=1, \ldots, N_{i}}$ with $M_{i} \geq C N_{i}$, such that $\left(b_{j}\right)_{j=1, \ldots, M_{i}}$ is a subset of

$$
P_{i}:=\left\{h_{i}+\sum_{j=1}^{q} r_{j} k_{j}^{(i)} \mid 0 \leq r_{j}<s_{j}^{(i)}\right\}
$$

with certain $h_{i}, k_{1}^{(i)}, \ldots, k_{q}^{(i)} \in \mathbb{Z}, s_{1}^{(i)}, \ldots, s_{q}^{(i)} \in \mathbb{N}$ and $s_{1}^{(i)} s_{2}^{(i)} \ldots s_{q}^{(i)} \leq K N_{i}$. Fix now any $i$, and for simplicity we omit the index $i$ in the above notations, i.e., we put $M:=M_{i}, h:=h_{i}$ and so on. In the sequel, we will put $K=1$ and $h=0$. The general case is treated similarly. Further, for $k=1, \ldots, M$, we set

$$
b_{k}=r_{1}^{(k)} k_{1}+\cdots+r_{q}^{(k)} k_{q}
$$

and we identify $b_{k}$ with the vector

$$
\left(r_{1}^{(k)}, \ldots, r_{q}^{(k)}\right)=: \boldsymbol{r}_{k}
$$

Consider now vectors

$$
\boldsymbol{u}:=\left(\begin{array}{c}
u_{1} \\
\vdots \\
u_{q}
\end{array}\right) \text {, }
$$

with $-\left(s_{j}-1\right) \leq u_{j} \leq\left(s_{j}-1\right)$ for $j=1, \ldots, q$. Let $\gamma:=\frac{C^{2}}{1+2^{q}}$, then it can be shown that there exist at least $\gamma N$ different vectors $\boldsymbol{u}$ such that there exist at least $\gamma N$ pairs $\boldsymbol{r}_{k}, \boldsymbol{r}_{l}$ with $\boldsymbol{r}_{k}-\boldsymbol{r}_{l}=\boldsymbol{u}$. In the sequel, we will refer to this observation as Property 1. Take now $\gamma N$ such $q$-tuples $\boldsymbol{u}$ having Property 1 and consider the corresponding $\gamma N$ values

$$
\left\{\left(u_{1} k_{1}+\cdots+u_{q} k_{q}\right) \boldsymbol{\alpha}\right\}, \quad \text { in }[0,1)^{2} .
$$


Let $L:=\frac{2}{\gamma}$, then there is a $\beta \in[0,1)$, such that the box $\left[\beta, \beta+\sqrt{\frac{L}{\gamma N}}\right)^{2}$ contains at least $L$ elements of the form (10), say the elements

$$
\left\{\left(u_{1}^{(x)} k_{1}+\cdots+u_{q}^{(x)} k_{q}\right) \boldsymbol{\alpha}\right\}, \quad \text { for } x=1, \ldots, L .
$$

We call this fact Property 2. Note that for sake of simplicity, we treat $\gamma N$ and $L$ as if they were integers. The general case can be handled analogously with some minor technical changes.

For every choice of $x$, we consider now $\gamma N$ pairs of $q$-tuples, say

$$
\boldsymbol{r}_{i, x}:=\left(\begin{array}{c}
r_{1}^{(i, x)} \\
\vdots \\
r_{q}^{(i, x)}
\end{array}\right) \text {, and } \tilde{\boldsymbol{r}}_{i, x}:=\left(\begin{array}{c}
\tilde{r}_{1}^{(i, x)} \\
\vdots \\
\tilde{r}_{q}^{(i, x)}
\end{array}\right) \text {, }
$$

for $i=1, \ldots, \gamma N$, such that

$$
\boldsymbol{r}_{i, x}-\tilde{\boldsymbol{r}}_{i, x}=\left(\begin{array}{c}
u_{1}^{(x)} \\
\vdots \\
u_{q}^{(x)}
\end{array}\right)
$$

We define, for $x=1, \ldots, L$, the sets

$$
\mathcal{M}_{x}:=\left\{\boldsymbol{r}_{i, x} \mid i=1, \ldots, \gamma N\right\}
$$

It can be proven that there exist $x, y \in\{1, \ldots, L\}$ with $x \neq y$ such that

$$
\left|\mathcal{M}_{x} \cap \mathcal{M}_{y}\right| \geq \frac{N}{L^{2}}
$$

Let now $x$ and $y$ satisfying (11) be given. Let

$$
\begin{aligned}
& \boldsymbol{r}_{i}, i=1, \ldots, \frac{N}{L^{2}}, \\
& \tilde{\boldsymbol{r}}_{i, x}, i=1, \ldots, \frac{N}{L^{2}}, \\
& \tilde{\boldsymbol{r}}_{i, y}, \quad i=1, \ldots, \frac{N}{L^{2}}
\end{aligned}
$$

be such that

$$
\boldsymbol{r}_{i}-\tilde{\boldsymbol{r}}_{i, x}=\left(\begin{array}{c}
u_{1}^{(x)} \\
\vdots \\
u_{q}^{(x)}
\end{array}\right), \text { and } \boldsymbol{r}_{i}-\tilde{\boldsymbol{r}}_{i, y}=\left(\begin{array}{c}
u_{1}^{(y)} \\
\vdots \\
u_{q}^{(y)}
\end{array}\right)
$$


Then,

$$
\tilde{\boldsymbol{r}}_{i, y}-\tilde{\boldsymbol{r}}_{i, x}=\left(\begin{array}{c}
u_{1}^{(x)}-u_{1}^{(y)} \\
\vdots \\
u_{q}^{(x)}-u_{q}^{(y)}
\end{array}\right)=:\left(\begin{array}{c}
z_{1} \\
\vdots \\
z_{q}
\end{array}\right) \text {, }
$$

for $i=1, \ldots, \frac{N}{L^{2}}$. Due to Property 2, we have

$$
\sqrt{\frac{L}{\gamma N}} \geq\left\|\left\{\left(z_{1} k_{1}+\cdots+z_{q} k_{q}\right) \boldsymbol{\alpha}\right\}\right\|_{\infty}
$$

To sum up, we have shown that for all $N_{i}$ there exist at least

$$
\frac{N_{i}}{L^{2}}=\frac{C^{4}}{4\left(1+2^{q}\right)^{2}} N_{i}=: \tau N_{i}
$$

pairs $(k, l)$ with $1 \leq k \neq l \leq N_{i}$, such that all expressions $\left\|\left\{a_{k} \boldsymbol{\alpha}\right\}-\left\{a_{l} \boldsymbol{\alpha}\right\}\right\|_{\infty}$ have the same value and satisfy

$$
\left\|\left\{a_{k} \boldsymbol{\alpha}\right\}-\left\{a_{l} \boldsymbol{\alpha}\right\}\right\|_{\infty} \leq \sqrt{\frac{L}{\gamma N_{i}}}=\sqrt{\frac{2\left(1+2^{q}\right)^{2}}{C^{4}}} \frac{1}{N_{i}^{1 / 2}}=: \psi \frac{1}{N_{i}^{1 / 2}} .
$$

Note, that $\tau<1$ and $\psi$ only depends on $d, q$ and $C$ (and on $K$ if $K \neq 1$ ). For every $i$ choose now $\psi_{i}$ minimal such that there exist at least $\tau N_{i}$ pairs $(k, l)$ with $1 \leq k \neq l \leq N_{i}$, such that

$$
\left\|\left\{a_{k} \boldsymbol{\alpha}\right\}-\left\{a_{l} \boldsymbol{\alpha}\right\}\right\|_{\infty}=\psi_{i} \frac{1}{N_{i}^{1 / 2}}
$$

Of course, $\psi_{i} \leq \psi$ for all $i$. Let now $\rho:=\frac{\tau^{1 / 2} C^{2}}{3\left(1+2^{q}\right)}$ (hence $\rho<\tau$ ) and assume that $\psi_{i}<\rho$ for infinitely many $i$. Therefore, we have for these $i$

$$
\begin{aligned}
& \frac{1}{N_{i}} \#\left\{1 \leq k \neq l \leq N_{i} \mid\left\|\left\{a_{k} \boldsymbol{\alpha}\right\}-\left\{a_{l} \boldsymbol{\alpha}\right\}\right\|_{\infty} \leq \rho \frac{1}{N_{i}^{1 / 2}}\right\} \\
& \geq \tau=\left(\frac{3 \rho\left(1+2^{q}\right)}{C^{2}}\right)^{2}>(2 \rho)^{2},
\end{aligned}
$$

and consequently the pair correlations of $\left(\left\{a_{n} \boldsymbol{\alpha}\right\}\right)_{n \in \mathbb{N}}$ cannot be Poissonian.

Assume now that $\psi_{i} \geq \rho$ for infinitely many $i$. Consequently, there exist $s_{1}, s_{2} \in$ $[\rho, \psi)$ such that

$$
\psi_{i} \in\left(s_{1}, s_{2}\right]
$$


for infinitely many $i$. In the following, we only consider these $i$ and we will set $s_{1}=\rho+a \frac{\tau C^{2}}{32 \sqrt{2}\left(1+2^{q}\right)}$ and $s_{2}:=\rho+(a+1) \frac{\tau C^{2}}{32 \sqrt{2}\left(1+2^{q}\right)}$, for some

$$
a \in\left\{0,1, \ldots,\left\lceil(\psi-\rho) \frac{32 \sqrt{2}\left(1+2^{q}\right)}{\tau C^{2}}\right\rceil\right\} .
$$

Then, we have, due to the fact that there are at least $\tau N_{i}$ pairs with distance equal to $\psi_{i} \frac{1}{N_{i}^{1 / 2}}$,

$$
\begin{aligned}
& \frac{1}{N_{i}} \#\left\{1 \leq k \neq l \leq N_{i} \mid\left\|\left\{a_{k} \boldsymbol{\alpha}\right\}-\left\{a_{l} \boldsymbol{\alpha}\right\}\right\|_{\infty} \leq s_{2} \frac{1}{N_{i}^{1 / 2}}\right\} \\
& \quad-\frac{1}{N_{i}} \#\left\{1 \leq k \neq l \leq N_{i} \mid\left\|\left\{a_{k} \boldsymbol{\alpha}\right\}-\left\{a_{l} \boldsymbol{\alpha}\right\}\right\|_{\infty} \leq s_{1} \frac{1}{N_{i}^{1 / 2}}\right\} \\
& \geq \tau .
\end{aligned}
$$

If $\left(\left\{a_{n} \alpha\right\}\right)_{n \in \mathbb{N}}$ were Poissonian, then the above difference should converge to

$$
4\left(s_{2}^{2}-s_{1}^{2}\right)=4\left(s_{2}+s_{1}\right)\left(s_{2}-s_{1}\right)<\tau
$$

as $i \rightarrow \infty$, which is a contradiction to the previous inequality.

Acknowledgements Open access funding provided by Johannes Kepler University Linz. We want to thank Christoph Aistleitner for helping to improve the statement of Theorem 2.

Funding Special Upper Austrian Grant.

Open Access This article is distributed under the terms of the Creative Commons Attribution 4.0 International License (http://creativecommons.org/licenses/by/4.0/), which permits unrestricted use, distribution, and reproduction in any medium, provided you give appropriate credit to the original author(s) and the source, provide a link to the Creative Commons license, and indicate if changes were made.

\section{Appendix: Proof of Corollary 1}

We again prove the result for $d=2$ only, as the general case is carried out quite analogously.

There exists a constant $\rho$ with $0<\rho<1$ having the following property: For every pair $\left(\alpha_{1}, \alpha_{2}\right)$ there exist infinitely many $q \in \mathbb{N}$ such that

$$
\max \left(\left\{q \alpha_{1}\right\},\left\{q \alpha_{2}\right\}\right)<\frac{\rho}{q^{1 / 2}},
$$

see, e.g., [7]. Consider now such a $q$ and set

$$
\frac{\theta}{q^{1 / 2}}=\max \left(\left\{q \alpha_{1}\right\},\left\{q \alpha_{2}\right\}\right),
$$


where $0<\theta \leq \rho$. Let $A=A(q)$ be the minimal integer such that

$$
\left(\left(\frac{1}{A \theta}\right)^{2 / 3}+1\right)^{3} \theta^{2}<\frac{1+\theta^{2}}{2}
$$

holds, which is possible due to $\theta \leq \rho<1$. Note that $A$ is the larger, the larger $\theta$ is. Hence, the values of $A$ are bounded by the value obtained for $\theta=\rho$. This $A$ will be denoted by $A_{\rho}$. Further, for the choice of $B=B(q):=\frac{2}{1+\theta^{2}}$, we have $B \geq \frac{2}{1+\rho^{2}}>1$. Choose $L=L(q):=\left\lceil\left(\frac{1}{A \theta}\right)^{2 / 3}\right\rceil$ and the real $\tilde{v}$ such that

$$
L \frac{\theta}{q^{1 / 2}}=\frac{1}{\left(A^{2} L q-\tilde{v}\right)^{1 / 2}},
$$

i.e.,

$$
\tilde{v}=A^{2} L q-\frac{q}{L^{2} \theta^{2}}
$$

In the sequel, we will show that

$$
\frac{q}{L^{2} \theta^{2}} \geq B L q
$$

and consequently,

$$
\tilde{v} \leq\left(A^{2}-B\right) L q
$$

Clearly, Eq. (13) is equivalent to $B L^{3} \theta^{2} \leq 1$. Now

$$
B L^{3} \theta^{2} \leq B\left(\left(\frac{1}{A \theta}\right)^{2 / 3}+1\right)^{3} \theta^{2}
$$

and hence, (13) holds if

$$
\left(\left(\frac{1}{A \theta}\right)^{2 / 3}+1\right)^{3} \theta^{2}<\frac{1}{B}=\frac{1+\theta^{2}}{2}
$$

which is true due to the definition of $A$ and $B$, respectively. Let $v:=\lfloor\tilde{v}\rfloor$ and $N:=$ $A^{2} L q-v$. Note, that by (14) we have $N \geq B L q$ and this, by the definition of $B$ and $L$ tends to infinity for $q$ to infinity.

We consider now the sequence elements $\boldsymbol{x}_{1}, \ldots, \boldsymbol{x}_{N=A^{2} L q-v}$, and study the distances of the pairs

$$
\left(\boldsymbol{x}_{1}, \boldsymbol{x}_{1+q L}\right),\left(\boldsymbol{x}_{2}, \boldsymbol{x}_{2+q L}\right), \ldots,\left(\boldsymbol{x}_{N-q L}, \boldsymbol{x}_{N}\right) .
$$


Using the estimate (14), we derive that there are

$$
\begin{aligned}
N-q L=\left(\left(A^{2}-1\right) L\right) q-v & \geq(B-1) L q \\
& \geq\left(\frac{2}{1+\rho^{2}}-1\right) L q \\
& \geq A^{2} L q\left(\frac{2}{1+\rho^{2}}-1\right) \frac{1}{A_{\rho}^{2}} \\
& \geq \gamma N
\end{aligned}
$$

such pairs, where $\gamma$ is a positive fixed constant independent on $q$.

Now, for each such pair, we get (for $k=1, \ldots, N-q L$ )

$$
\begin{aligned}
\left\|\boldsymbol{x}_{k}-\boldsymbol{x}_{k+q L}\right\|_{\infty} & =\max \left(\left\{L q \alpha_{1}\right\},\left\{L q \alpha_{2}\right\}\right) \\
& =L \frac{\theta}{q^{1 / 2}} .
\end{aligned}
$$

The second equality is true due to the following inequality

$$
\frac{1}{\sqrt{N}} \leq L \frac{\theta}{q^{1 / 2}} \leq \frac{3}{\sqrt{N}},
$$

which we will prove subsequently. First, note that we have

$$
\begin{aligned}
0 & <\sqrt{N}\left(\frac{1}{\left(A^{2} L q-\tilde{v}\right)^{1 / 2}}-\frac{1}{\left(A^{2} L q-v\right)^{1 / 2}}\right) \\
& =\left(\frac{A^{2} L q-v}{A^{2} L q-\tilde{v}}\right)^{1 / 2}-1=\left(1+\frac{\tilde{v}-v}{\left(A^{2} L q-\tilde{v}\right)}\right)^{1 / 2}-1 \\
& <\left(1+\frac{1}{B L q}\right)^{1 / 2} \leq\left(1+\frac{1}{q}\right)^{1 / 2}<2,
\end{aligned}
$$

where we used the estimate (14) for $\tilde{v}$. Further, due to above estimate and (12), we also have

$$
\begin{aligned}
0 & <\sqrt{N}\left(L \frac{\theta}{q^{1 / 2}}-\frac{1}{\sqrt{N}}\right) \\
& =\sqrt{N}\left(\frac{1}{\left(A^{2} L q-\tilde{v}\right)^{1 / 2}}-\frac{1}{\left(A^{2} L q-v\right)^{1 / 2}}\right) \\
& <2,
\end{aligned}
$$

i.e.,

$$
\frac{1}{\sqrt{N}} \leq L \frac{\theta}{q^{1 / 2}} \leq \frac{3}{\sqrt{N}}
$$


As the inequality from above is valid for infinitely many $q$ and as a consequence thereof, there exists an $a \in\left\{0,1, \ldots,\left\lceil 3 \frac{100}{\gamma}\right\rceil\right\}$ such that

$$
\frac{1+a \frac{\gamma}{100}}{\sqrt{N}} \leq L \frac{\theta}{q^{1 / 2}} \leq \frac{1+(a+1) \frac{\gamma}{100}}{\sqrt{N}}
$$

for infinitely many $q$.

In the following, we consider a sequence $\left(q_{l}\right)$ of such $q$ with corresponding $\left(N_{l}\right)$ and define

$$
\begin{aligned}
& s_{1}:=1+a \frac{\gamma}{100} \\
& s_{2}:=1+(a+1) \frac{\gamma}{100} .
\end{aligned}
$$

Assume that $\left(\boldsymbol{x}_{n}\right)_{n \in \mathbb{N}}$ were Poissonian, then we had

$$
\lim _{l \rightarrow \infty} \Gamma_{i}(l):=\lim _{l \rightarrow \infty} \frac{1}{N_{l}} \#\left\{1 \leq l \neq m \leq N_{i}:\left\|\boldsymbol{x}_{l}-\boldsymbol{x}_{m}\right\|_{\infty} \leq \frac{s_{i}}{N^{1 / 2}}\right\}=4 s_{i}^{2},
$$

for $i=1,2$.

But $\Gamma_{2}(l) \geq \Gamma_{1}(l)+\gamma$ due to (15), which gives a contradiction as we also have

$$
4 s_{2}^{2}-4 s_{1}^{2}=4\left(s_{2}+s_{1}\right)\left(s_{2}-s_{1}\right) \leq 32 \frac{\gamma}{100} .
$$

\section{References}

1. Aichinger, I., Aistleitner, C., Larcher, G.: On quasi-energy-spectra, pair correlations of sequences and additive combinatorics. In: Dick, J., Kuo, F.Y., Woźniakowski, H. (eds.) Contemporary Computational Mathematics - Celebration of the 80th Birthday of Ian Sloan. Springer, Cham (2018)

2. Aistleitner, C., Lachmann, T., Pausinger, F.: Pair correlations and equidistribution. J. Number Theory 182, 206-220 (2018)

3. Aistleitner, C., Lachmann, T., Technau, N.: There is no Khintchine threshold for metric pair correlations. arXiv:1802.02659 (2018)

4. Aistleitner, C., Larcher, G., Lewko, M.: Additive energy and the Hausdorff dimension of the exceptional set in metric pair correlation problems. With an appendix by Jean Bourgain. Israel J. Math. 222(1), 463-485 (2017)

5. Bloom, T.F., Walker, A.: GCD sums and sum-product estimates. arXiv:1806.07849 (2018)

6. Bondarenko, A., Seip, K.: GCD sums and complete sets of square-free numbers. Bull. Lond. Math. Soc. 47(1), 29-41 (2015)

7. Bugeaud, Y.: Distribution Modulo 1 and Diophantine Approximation. Cambridge University Press, Cambridge (2012)

8. El-Baz, D., Marklof, J., Vinogradov, I.: The two-point correlation function of the fractional parts of $\sqrt{n}$ is Poisson. Proc. Am. Math. Soc. 143(7), 2815-2828 (2015)

9. El-Baz, D., Marklof, J., Vinogradov, I.: The distribution of directions in an affine lattice: two-point correlations and mixed moments. Int. Math. Res. Not. 2015, 1371-1400 (2015)

10. Gál, I.S.: A theorem concerning diophantine approximations. Nieuw Arch. Wiskd. 2(23), 13-38 (1949) 
11. Grepstad, S., Larcher, G.: On pair correlation and discrepancy. Arch. Math. 109, 143-149 (2017)

12. Heath-Brown, D.R.: Pair correlation for fractional parts of $\alpha n^{2}$. Math. Proc. Camb. Philos. Soc. 148(3), 385-407 (2010)

13. Kuipers, L., Niederreiter, H.: Uniform Distribution of Sequences. Wiley, New York (1974)

14. Larcher, G., Stockinger, W.: Pair correlation of sequences $\left(\left\{a_{n} \alpha\right\}\right)_{n \in \mathbb{N}}$ with maximal order of additive energy. Accepted by Math. Proc. of Cambridge Philosophical Society. arXiv:1802.02901 (2018)

15. Larcher, G., Stockinger, W.: Some negative results related to Poissonian pair correlation problems. arXiv:1803.052361 (2018)

16. Marklof, J.: Distribution modulo one and Ratners theorem. In: Equidistribution in number theory, an introduction. NATO Sci. Ser. II Math. Phys. Chem., vol. 237, pp. 217-244. Springer, Dordrecht (2007)

17. Nair, R., Pollicott, M.: Pair correlations of sequences in higher dimensions. Israel J. Math. 157, 219-238 (2007)

18. Rudnick, Z., Zaharescu, A.: A metric result on the pair correlation of fractional parts of sequences. Acta Arith. 89(3), 283-293 (1999)

19. Rudnick, Z., Sarnak, P., Zaharescu, A.: The distribution of spacings between the fractional parts of $n^{2} \alpha$. Invent. Math. 145(1), 37-57 (2001)

20. Rudnick, Z., Sarnak, P.: The pair correlation function of fractional parts of polynomials. Commun. Math. Phys. 194(1), 61-70 (1998)

21. Steinerberger, S.: Localized quantitative criteria for equidistribution. Acta Arith. 180, 183-199 (2017)

22. Walker, A.: The primes are not metric Poissonian. Mathematika 64, 230-236 (2018)

Publisher's Note Springer Nature remains neutral with regard to jurisdictional claims in published maps and institutional affiliations.

\section{Affiliations}

\section{Aicke Hinrichs ${ }^{2}$. Lisa Kaltenböck ${ }^{1}$. Gerhard Larcher ${ }^{1}$. Wolfgang Stockinger ${ }^{3}$ (D) Mario Ullrich ${ }^{2}$}

Aicke Hinrichs

aicke.hinrichs@jku.at

Lisa Kaltenböck

lisa.kaltenboeck@jku.at

Gerhard Larcher

gerhard.larcher@jku.at

Mario Ullrich

mario.ullrich@jku.at

1 Institut für Finanzmathematik und Angewandte Zahlentheorie, Johannes Kepler Universität Linz, Altenbergerstraße 69, 4040 Linz, Austria

2 Institut für Analysis, Johannes Kepler Universität Linz, Altenbergerstraße 69, 4040 Linz, Austria

3 University of Oxford, Andrew Wiles Building, Radcliffe Observatory Quarter, Woodstock Road, Oxford OX2 6GG, UK 